\title{
Milademetan Tosylate
}

National Cancer Institute

\section{Source}

National Cancer Institute. Milademetan Tosylate. NCI Thesaurus. Code C107384.

The tosylate form of milademetan, an orally available MDM2 (murine double minute 2) antagonist with potential antineoplastic activity. Upon oral administration, milademetan binds to, and prevents the binding of MDM2 protein to the transcriptional activation domain of the tumor suppressor protein p53. By preventing this MDM2-p53 interaction, the proteasome-mediated enzymatic degradation of p53 is inhibited and the transcriptional activity of p53 is restored. This results in the restoration of p53 signaling and leads to the p53-mediated induction of tumor cell apoptosis. MDM2, a zinc finger protein and a negative regulator of the p53 pathway, is overexpressed in cancer cells; it has been implicated in cancer cell proliferation and survival. 